\title{
Cue Now, Reflect Later: A Study of Delayed Reflection of Diary Events
}

\author{
Ming Ki Chong ${ }^{1}$, Jon Whittle ${ }^{1}$, Umar Rashid ${ }^{2}$ and Chee Siang Ang ${ }^{2}$ \\ ${ }^{1}$ School of Computing \& Communications, InfoLab21, Lancaster University, \\ Lancaster, LA1 4WA, United Kingdom \\ ${ }^{2}$ School of Engineering \& Digital Arts, Jennison Building, University of Kent, \\ Canterbury, CT2 7NT, United Kingdom \\ mingki@acm.org, j.n.whittledlancaster.ac.uk, \\ \{U.R.Mir,C.S.Ang\}@ kent.ac.uk
}

\begin{abstract}
Diary studies require participants to record entries at the moment of events, but the process often distracts the participants and disrupts the flow of the events. In this work, we explore the notion of delayed reflection for diary studies. Users quickly denote cues of diary events and only reflect on the cues later when they are not busy. To minimize disruptions, we employed a squeeze gesture that is swift and discreet for denoting cues. We investigated the feasibility of delayed reflection and compared it against a conventional digital diary that requires users to reflect immediately at the time of entry. In a weeklong field study, we asked participants to record their daily experiences with both types of diaries. Our results show that users' preference is context-dependent. Delayed reflection is favored for use in contexts when interruptions are deemed inappropriate (e.g. in meetings or lectures) or when the users are mobile (e.g. walking). In contrast, the users prefer immediate reflection when they are alone, such as during leisure and downtime.
\end{abstract}

Keywords: Diary Study; Delayed Reflection, SqueezeDiary

\section{Introduction}

Field studies that require the full-time presence of researchers are impractical to conduct. Researchers have therefore adopted the diary method as an alternative for recording everyday behaviors of participants [11]. The method relies on participants to capture events in situ as they happen, so it requires no researcher presence and the participants provide first-hand self-reporting of their experience. Two types of diary studies exist [2]: feedback studies where participants answer predefined questions about events, and elicitation studies where participants capture media that are used as prompts for discussion in interviews.

Feedback studies enable researchers to capture immediate answers from participants. For example, in a study of understanding task switching and interruptions in the work place, Czerwinski et al. [5] asked participants to reflect while they were working. This form of immediate reflection provides the benefit that diary data is not influenced by memory decay. However, the immediate reflection distracts participants from carrying out their main task [2]. 

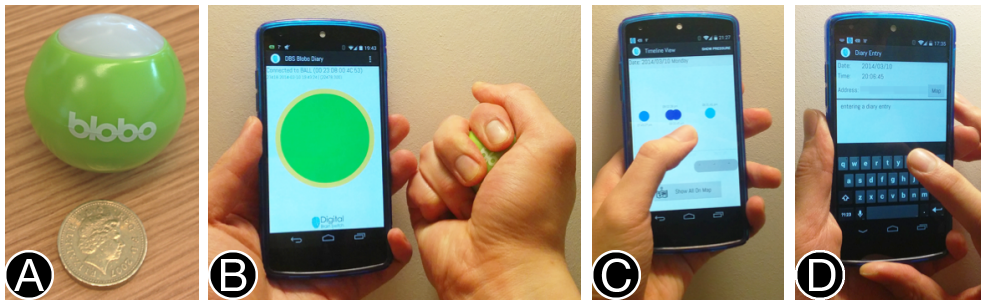

Fig. 1. Interaction of the delayed reflection diary used in our study. (A) The squeeze sensor. (B) Squeeze to record a cue. (C) Review recorded event cues. (D) Diary entry. (Source: [3])

In elicitation studies, interviews are conducted at the end of the study. Detailed information may therefore be lost due to prolonged delay, and participants' emotional responses may change over a period of time [8]. To help participants reconstruct their memories, researchers have investigated the role of different media (e.g. photographs, audio recordings, location information, and tangible artifacts) in diary studies [2] and have used lifelogging tools to support recall and reflection $[6,9,12]$.

Researchers have proposed an alternative elicitation method for conducting diary studies on mobile phones [1]. The intention is to lower users' burden by allowing them to only capture "snippets of information" via text (SMS), voicemail or pictures (MMS). The users then explicitly upload the snippets onto a server for revisiting later. The snippets serve as prompts for users to complete full diary entries at a later, more convenient time. We embrace this notion of delayed reflection: events are first triggered and stored as cues, and reflection is carried out during users' downtime.

We adopted SqueezeDiary, a delayed reflection diary tool that uses squeeze gestures for triggering event cues $[3,4]$. The application has several advantages. Squeeze is simple and quick to perform. The small form factor of the tactile sensor (see Fig. 1) enables users to squeeze it swiftly without the need for accessing the smartphone. Data is stored locally, so the system requires no explicit uploading of information.

In this paper, we present a comparative study between delayed reflection for elicitation and a conventional digital text diary that requires immediate feedback from users. We first present the implementation of the two diaries, and then report on our weeklong deployment of the diaries. The results reveal that users' preference in the type of diary is context-dependent. Participants reported that they would prefer to use delayed reflection for recording events when they are mobile (e.g. walking), in a social event (e.g. meeting with colleagues), or in a restricted context (e.g. attending a lecture). However, the participants would prefer to use the conventional text diary during their leisure and downtime. In this case, they can reflect and enter diary entries straightaway, so the process of triggering an event instance before entering a diary description becomes unnecessary.

\section{Diary Applications}

To learn the differences between immediate reflection versus delayed reflection, we studied the use of two digital diaries: a conventional digital text diary for immedi- 


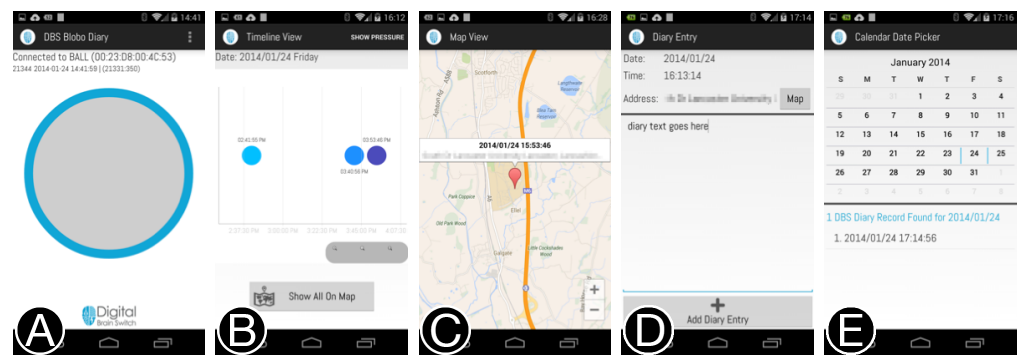

Fig. 2. Screenshots of SqueezeDiary, an implementation of delayed reflection.

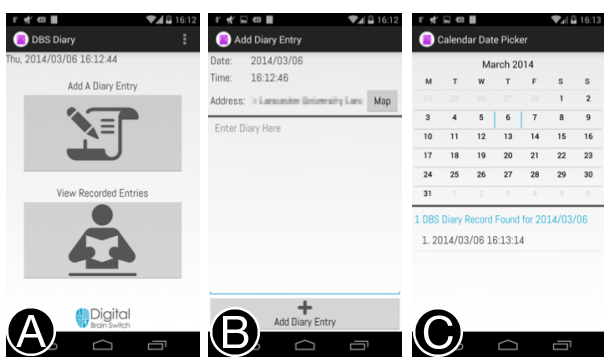

Fig. 3. Screenshots of text diary, an implementation of immediate reflection.

ate feedback and a delayed reflection diary. Both applications are designed for recording life events/experiences that users want to keep a record of. We implemented and tested our prototypes on Nexus 4/5 smartphones running Android version 4.4.2.

We adopted SqueezeDiary $[3,4]$ as an application for delayed reflection (see Fig. 2). A user first squeezes a sensor to trigger the system to record an event instance, and the user later enters a description for the event retrospectively. The application uses a small tactile ball as a squeezable user interface, and it interfaces with the smartphone through Bluetooth. The sensor device can be carried in a pocket, and users can squeeze it easily when needed, without accessing their smartphone. A user triggers an event instance by simply squeezing the sensor for two seconds, and the system automatically $\operatorname{logs}$ the location, the timestamp and the pressure levels. Our application provides visualizations of the recorded events on a timeline (2B) and on a map (2C). The application initially provides a graphical visualization of squeeze pressure, but we abandon it as a pre-study revealed that the pressure visualization provides no help for users to recall detail memory events. The application included an interface for users to add retrospective descriptions for their recorded events (2D), and a calendar interface for selecting recorded diary entries (2E).

For immediate reflection, users provide a textual description when they add a diary entry. We implemented a simplified diary application that has only two UI options (see Fig. 3). One option is for adding a new diary entry that automatically includes GPS location and timestamp (3B). The information serves as temporal and location cues to help the users to reconstruct their episodic memory [6,9]. The second UI option allows users to revisit and update their records (3C). 


\section{Diary Study}

We conducted a weeklong field trial to compare the two diaries. We recruited 8 participants (3F), aged between 19 and $27(M=22.25, S D=3.20)$. The participants were students at our institutions, from a variety of academic disciplines. They all owned a smartphone (either Android or iPhone) and self-rated as proficient smartphone users.

In the briefing we demonstrated the operations of both diary applications and also asked the participants to practice using the applications by recording sample diary entries. We explained to the participants that their task was to record instances of when they realized that they were not focusing or struggling to concentrate on their current task; for example, doing one thing but thinking about another. We selected this task because our target domains are well-being related applications, and the problem of 'doing one thing but thinking about another' is one representative of such applications. At the end of the briefing session, we gave the devices to the participants and instructed them to start recording on the next day. We also provided demonstration videos in case the participants encountered problems with using the diaries.

The recording lasted for six days. The study followed a within-group study design; half of the participants started with the squeeze diary and then switched to the text diary after recording for three days, and vice versa for the other half. During the recording period, the participants used the diary for the entire day; they started recording from the point they awoke until they retired in the evening. We instructed the participants to record instances only during weekdays when they were usually busy. To keep the participants engaged with the study, we sent an email reminder every evening [10].

After recording for six days, we invited the participants back for exit interviews; each session lasted for approximately 30 minutes. We used the diary entries to prompt the participants on how they used the diary applications; we also elicited qualitative feedback, as well as suggestions for improvement.

\section{$4 \quad$ Results}

In total, our participants recorded 120 delayed-reflection diary entries $(M=15$, $S D=10.70, M d n=11.5)$ and 84 immediate-reflection diary entries $(M=10.5, S D=6.65$, $M d n=9.5)$. A Wilcoxon Signed-Rank Test did not reveal a significant difference in the number of entries between the two diaries $(N(8), Z=-1.47, p>0.05)$. Two members of our research team analyzed the collected data independently.

\subsection{Reflection: Immediate vs. Delayed}

We observed two types of users. One type of users found it easier to perform delayed reflection, as it allows them to continue with their primary task with minimum disruption. These users enjoyed the freedom of entering diary entries "at [their] own pace". Some participants mentioned that they occasionally found it "challenging to reflect" on an event if it was recorded after a lengthy period or if they had a busy day. 
For example, there was a day that a participant was busy for four consecutive hours (attending lectures and meetings), and she had squeezed the sensor several times. During her delayed reflection, a cluster of events was shown on the timeline which confused her. She clarified by examining the locations of the events, which helped her to differentiate whether she was in a lecture or in a meeting. Our participants described that seeing a cluster of events on a timeline made them realize how much they struggled to concentrate in specific events (e.g. attending "a boring lecture").

The second type of users that we observed preferred immediate reflection. These users could "only remember the gist" but forget the details quickly if the description was not recorded straightaway. One participant who had several part-time jobs mentioned that on a busy day he had to multitask frequently, so memories could overlap and detailed information could become vague if he did not record it immediately.

Our participants mentioned that when using the SqueezeDiary, they wrote a description immediately after they squeezed the sensor during their leisure, such as relaxing at home in the evening. In contrast, one participant used the mobile text diary for delayed reflection. He explained that he often switched his thoughts when he was changing venues between lectures, so he would not have enough time to reflect immediately and enter a description while in transit. He instead first started a diary entry with empty content, and then entered a detailed description later using the update feature.

\subsection{Context Preferences}

The participants preferred to use delayed reflection diary during busy working hours and in situations where using a phone was forbidden or considered inappropriate. The participants considered short notifications as acceptable (e.g. short vibrations or audio alerts on the phone for when an event cue is recorded), as it is similar to "receiving a text" message. However, taking out a phone and entering text would be deemed socially impolite (e.g. "rude to enter text in front of friends") or inappropriate during classes and lectures. The participants reported that they usually reflect on recorded events during their breaks (e.g. long breaks between lectures or lunchtime) and in the evening.

The participants also reported that they prefer to perform immediate reflection during their leisure or downtime (e.g. resting at home), when they were not bounded by work or duties, which gave them opportunities to self-reflect. The participants also mentioned that they preferred to write diaries when they were alone, as this eliminates distractions and social awkwardness. Some participants mentioned that if they were not busy it is more convenient to "just enter diaries", without needing to first squeeze a sensor to cue an event.

Overall, our participants disliked writing diary entries when they were moving. We learned that delayed reflection is good for recording instance of events during users' busy periods, while immediate reflection is suitable for leisure situations, as the action of first cueing an event before recording a description becomes unnecessary. 


\subsection{Visualization}

Our participants explained that the act of squeezing prompted them to consciously remember particular thoughts, and later seeing the cues of the recorded events helped them to recall those thoughts. Visualizing the cues on a timeline and on a map helped the participants to disambiguate events. They did this by correlating their daily schedules with the visualizations.

\subsection{User Feedback}

We also elicited general feedback on usability, alternative uses, and suggestions for improvement. One participant said that he kept the tactile squeeze ball in his trousers pocket. The "bulginess" reminded him to squeeze for an event. This indicates that the physicality of the sensor can act as a reminder for users to record events.

The participants reported several issues of SqueezeDiary. On several occasions users could not record due to unstable Bluetooth connection between the sensor and the smartphone. Once a connection was lost, the users needed to reconnect the devices manually. The participants suggested automatic reconnection. Battery life is another issue: leaving the sensor connected could deplete the battery overnight, which required the users to recharge the sensor when they had the opportunity during the next day. The sensor occasionally recorded false data when it was left inside a bag or in a pocket. All of these indicate that the performance of the sensor is very important. Users are sensitive to inaccurate data; false positives or missing data could render the diary ineffective. This could be explained that people use the recorded cues for assisting their recall of memory events, so some information is already embedded in their memory, which makes spotting inconsistency easy.

Suggestions include using the diaries as a reminder for tracking daily tasks. The time and location cues could improve prospective memory [13]. Suggestions for other memory aids include adding pictures and replacing text with voice narration, but some people reported that they do not like to listen to their own voices.

\subsection{Post-Study Impact}

After completing our study, two participants expressed that by reflecting on their recorded entries, they realized that they needed a better management strategy for multitasking. One participant noticed how much time she spent thinking about her work. Even during her downtime, she was constantly worrying about work. For instance, her diary entries include "I couldn't sleep ... thinking about how much work I need to do" (recorded at 4:24 am); and "packing my bags for a weekend away and thinking about whether I should with all my work". She explained that going through the diaries made her realize that she was consumed by her work and needed a break. Similarly, another participant also reported that seeing her own diaries made her "realize how stressed out [she was]". 


\section{Discussion}

From the study we learned that delayed reflection is a suitable method for people to record diaries when they are busy or in situations when they could not reflect immediately. However, there exists a trade-off of detailed information for users' convenience. Detailed information could be lost because people's memory decays due to interference over time, especially when they experience a busy day or reflect after a long period. Our study showed that location and temporal cues helped users disambiguate events; however, other studies have shown that these cues are only inferential, and they are insufficient for reconstruction of finer details [9]. For recalls of detailed memories, media elicitation techniques (e.g. recording visual cues) could be usefully combined. One way for this is to incorporate the diary application with a lifelogging camera (e.g. SenseCam [7]). The event instances could then be synchronized with the camera data to provide rich media information to help the users reflect.

Our study revealed that people have different preferences between immediate and delayed reflection. We were surprised that our participants found novel ways to use our diary applications as the other type of diary (e.g. using the update feature of text diary for delayed reflection). Hence, there is no one method that fits all; designers should consider creating diary applications that allow users to do both types of reflection, and let users decide their preferred usage.

People often reflect while they were traveling, in transit between venues, and in traffic. We should design interfaces that allow people to easily record thoughts when they are on the move. For instance, a participant mentioned that accessing his mobile phone to record a thought while riding a motorbike would be impossible. He would prefer to have sensors embedded into his gloves and use voice for recording descriptions. With the proliferation of wearable devices, we envision that in the near future we could use body-worn devices to detect users' intents to record diary events.

While automated recording devices (e.g. lifelogging cameras) generate overabundance of information, our dairy enables users to choose the moment they want to record, and the users can do it discretely without comprising privacy.

The gesture for triggering event instances could easily be extended with multiple gestures for new interactions. For example, users can customize a particular gesture for work related entries and another gesture for family related entries, so event annotation could be done at the time of recording. Instead of using a squeeze ball, the system could adopt other devices, like a smartwatch that senses user gesture (e.g. tapping), to record event instances. Also, delayed reflection could be applied beyond the diary study method. The diary application has other use cases. For example, it could be applied as a site-tracking tool for people to keep track of where they have visited. This is particularly convenient for tourists who enjoy exploring new places. They can mark down any interesting places and later use the recorded cues to reflect on their journey.

Finally, our study recruited university students as participants; their daily activities mainly involved attending lectures and societal meetings. It would be beneficial to study whether delayed reflection helps workers (e.g. office employees) to reflect on their busy activities and find ways to improve their work-life balance. 


\section{Conclusion}

This paper presented a weeklong in situ diary study that compared immediate reflection with delayed reflection. Our results show that people's preference is contextdependent. They preferred recording diary entries with delayed reflection during busy hours or when they cannot access their devices to provide a complete diary entry; however, the method became unnecessary during people's leisure when they are relaxed and alone.

\section{$7 \quad$ Acknowledgement}

This work is part of the Digital Brain Switch project and is funded by the EPSRC $(\mathrm{EP} / \mathrm{K} 025201 / 1)$.

\section{References}

1. Brandt, J., Weiss, N., Klemmer, S. R.: Txt 4 18r: Lowering the burden for diary studies under mobile conditions. In: Proc. CHI EA 2007, pp. 2303-2308, ACM, (2007)

2. Carter, S., and Mankoff, J.: When participants do the capturing: The role of media in diary studies. In Proc. CHI 2005, pp. 899-908, ACM, (2005)

3. Chong, M. K., Whittle, J., Rashid, U., and Ang, S. A.: Squeeze the Moment: Denoting Diary Events by Squeezing. In: Proc. UbiComp 2014 Adjunct, pp. 219-222, ACM, (2014)

4. Chong, M. K., Whittle, J., Rashid, U., and Ang, S. A.: SqueezeDiary: Using Squeeze Gesture as Triggers of Diary Events. In: Proc. MobileHCI 2014, pp. 427-429, ACM, (2014)

5. Czerwinski, M., Horvitz, E., and Wilhite, S.: A diary study of task switching and interruptions. In Proc. CHI 2004, pp. 175-182, ACM, (2004)

6. Gouveia, R., and Karapanos, E.: Footprint tracker: Supporting diary studies with lifelogging. In Proc. CHI 2013, pp. 2921-2930, ACM, (2013)

7. Hodges, S., Williams, L., Berry, E., Izadi, S., Srinivasan, J., Butler, A., Smyth, G., Kapur, N., and Wood, K.: Sensecam: A retrospective memory aid. In Proc. UbiComp 2006, pp. 177-193, Springer-Verlag (2006)

8. Isaacs, E., Konrad, A., Walendowski, A., Lennig, T., Hollis, V., and Whittaker, S.: Echoes from the past: How technology mediated reflection improves well-being. In Proc. CHI 2013, pp. 1071-1080, ACM, (2013)

9. Kalnikaite, V., Sellen, A., Whittaker, S., and Kirk, D.: Now let me see where i was: Understanding how lifelogs mediate memory. In Proc. CHI 2010, pp. 2045-2054, ACM, (2010)

10. Palen, L., and Salzman, M.: Voice-mail diary studies for naturalistic data capture under mobile conditions. In Proc. CSCW 2002, pp. 87-95, ACM, (2002)

11. Rieman, J.: The diary study: A workplace-oriented research tool to guide laboratory efforts. In INTERCHI 1993, pp. 321-326, ACM, (1993)

12. Sellen, A. J., Fogg, A., Aitken, M., Hodges, S., Rother, C., and Wood, K.: Do life-logging technologies support memory for the past?: An experimental study using sensecam. In Proc. CHI 2007, pp. 81-90, ACM, (2007)

13. Sellen, A. J., Louie, G., Harris, J. E., and Wilkins, A. J.: What brings intentions to mind? an in situ study of prospective memory. Memory 5, 4 (1997), pp. 483-507. 